\title{
Problemas metodológicos en el estudio de la cobertura informativa de los medios en los procesos electorales. Hacia una validación de la muestra.
}

\author{
Francisco de Jesús Aceves González*
}

\section{Introducción}

Hace algunos años, José Carlos Lozano (1994) en una acuciosa revisión sobre el status que guardaba el "análisis de contenido" en la práctica de la investigación de comunicación en América Latina, apuntaba como una de sus más serias limitaciones -el "talón de Aquiles"de esta herramienta metodológica, el problema relacionado con el tamaño de la muestra y su validación. Específicamente, se refería, al problema de la muestra necesaria para hacer generalizables los hallazgos obtenidos en un análisis de contenido sobre la cobertura informativa a los diversos partidos en un período electoral y afirmaba, que no existían evidencias de un estudio semejante en América Latina.

En efecto, en los trabajos publicados sobre la cobertura informativa de los medios en las contiendas electorales hasta el año 2000 (Aceves, 2000), que basan sus resultados en la técnica del análisis de contenido, el problema de la validación de la muestra, no se abordaba específicamente. Como se expondrá en el siguiente apartado, la determinación del tamaño de la muestra se definía de acuerdo ya a los intereses ya a las posibilidades (físicas y financieras) de cada investigador, y aunque algunos de ellos ajustaban, o trataban de ajustar la integración de su muestra de acuerdo a los criterios sugeridos por Stempel en 1952, en el sentido de que para analizar el contenido informativo de un periódico a lo largo de un año, bastaba con doce números del mismo y que "el aumento del tamaño de la muestra más allá de doce no producía resultados significativamente más precisos" (Krippendorff, 1990), así como la utilización de la "semana compuesta", cuyos resultados son analizadas por Riffe, Aust y Lacy publicado en su trabajo en 1993, en el que señalan que "dos semanas compuestas ofrecerían estimaciones confiables sobre las noticias locales para todo un año de ediciones periodísticas" (citado por Lozano, 1994). Sin embargo, la validación que tales muestras podrían tener para el caso de la prensa norteamericana, no necesariamente puede aplicarse para la situación mexicana. Pero además, ambos estudios contemplan la cobertura informativa, tomando como base

* Universidad de Guadalajara 
las "materias tratadas" o las "noticias locales", que los diarios realizan a lo largo de un año, es decir de un período que no abarca en sentido estricto, el comportamiento informativo que la prensa observa en el contexto de un proceso electoral.

La validación del tamaño muestral implica la realización de un análisis de contenido censal que abarque la totalidad del universo a investigar. Como lo sugiere Lozano, una vez obtenidos, mediante el censo, los datos totales del universo, se puede proceder a comparar los resultados obtenidos por distintos tamaños de muestra, con los resultados del censo y así determinar su confiabilidad estadística, es decir su validación.

La oportunidad de realizar un censo de estas características, se presentó a quien esto escribe, al ser invitado a coordinar la ejecución de un monitoreo sobre la cobertura informativa de las elecciones estatales del año 2000 en Jalisco, bajo los auspicios del Consejo Electoral del Estado de Jalisco y de la Universidad de Guadalajara. Los resultados obtenidos de esa investigación, son los que proporcionan el sustento empírico sobre el que se edifica la presente propuesta.

\section{Los estudios sobre análisis de contenido de la cobertura informativa de los procesos electorales}

En su estudio pionero sobre la investigación de la cobertura informativa de las campañas electorales, Petra Secanella (1983) realizó el análisis del espacio informativo que los diarios Excelsior, El Universal y Uno más Uno otorgaron a las campañas de los candidatos presidenciales en las contiendas electorales de 1970, 1976 y 1982, delimitó el tamaño de su muestra en los últimos diez días del mes de mayo del año respectivo. Las unidades de análisis que consideró fueron "las menciones en primera página, columnas de noticias e ilustraciones". Vale la pena apuntar que en dichas fechas, el período destinado a las campañas electorales abarcaba un promedio de ocho meses, que iniciaban con el destape del candidato del partido oficial y concluían con la jornada electoral. Durante ese tiempo, los medios reportaban el recorrido litúrgico, que el nuevo ungido realizaba a lo largo del país. No importaba que lo hiciera sin adversario al frente como el caso de López Portillo en 1976. El sentimiento de inutilidad de tales procesos se condensa en la trase lapidaria de Rafacl Segovia 
Si nuestras elecciones son comicios sin misterio, sin angustias finales. Si todo el país sabe que el candidato del PRI es el próximo presidente de la República, resulta ocioso y contraproducente obligarlo a desgastarse durante ocho meses que bien considerados y mejor aprovechados podrían reducirse a dos (citado por Secanella, 1983: 154)

La competencia en las contiendas electorales en México inicia con el polémico proceso de 1988. Coincidentemente, aparecen en el campo investigativo de la comunicación, diversos estudios enfocados a la cobertura informativa de los medios que utilizan en forma harto ecléctica, por decirlo de alguna manera, el análisis de contenido.

La prensa editada en la capital del país, de circulación nacional, es objeto de diversos estudios. Adrián de Garay y Laura Moya (1989) analizan la cobertura de seis diarios (El Universal, El Día, El Financiero, Excelsior, La Jornada y el Uno más Uno) a las campañas presidenciales. Aunque sin presentar una definición específica sobre su procedimiento metodológico, los autores señalan que sus resultados están basados en el "seguimiento" realizado de los meses de enero a junio, es decir en un censo de las ediciones correspondientes a dicho período. Como unidad de análisis definieron a las "columnas", a las cuales se asignó un valor igual, sin tomar en cuenta las diferencias en el formato de los diarios analizados. Por su parte Raúl Trejo Delarbre (1990) que analizó los diarios Excelsior, El Universal, Novedades, El Nacional, La Jornada y el Uno más Uno, especificó que la selección de su muestra "dependió de las fechas que, al azar, estableció Pablo Arredondo... para su estudio sobre noticiarios televisivos". La muestra abarcaba un total de 26 días del 23 de marzo al 18 de agosto ( 16 de los cuales correspondían al proceso electoral y los 10 restantes a los conflictos poselectorales). Como unidades de análisis se consideraron las notas informativas (excluyendo géneros de opinión), las fotografías sobre las actividades de los candidatos en campaña y las gacetillas (inserciones pagadas que son editados como material informativo).

Por su parte, Gilberto Fregoso (1990) selecciona para su análisis de la cobertura electoral de 1988 a los tres diarios de mayor circulación en el área Metropolitana de Guadalajara (El Informador, El Occidental y Ocho Columnas. En su estudio, Fregoso delimita su muestra en 66 ejemplares por cada diario. Para su confección "se tomó una muestra 
al azar que incluyó entre cuatro y seis números de cada uno por mes, durante el lapso de un año, comprendido desde (1) septiembre de 1987 a (18) agosto de 1988". De los días seleccionados 56 correspondían al período propiamente electoral y 10 eran fechas posteriores al 6 de julio. Como unidades de análisis determinó el conjunto de la información periodística, incluyendo las inserciones pagadas, y como unidades de medida utilizó el espacio $\left(\mathrm{cms}^{2}\right)$ y su equivalente en número de páginas.

El estudio sobre la cobertura informativa de los principales noticiarios televisivos (24 Horas y Día a Día) en las elecciones de 1988 (Arredondo, 1990) define una muestra de "55 días seleccionados al azar, entre septiembre de 1987 y agosto de 1988". Como en los casos anteriores, aquí también solamente 45 días del total de la muestra corresponden al período de las campañas electorales. La unidad de análisis es la noticia, de las cuales se registra su número y el espacio otorgado a las mismas.

Pero además de las elecciones presidenciales de 1988, Fregoso analiza ese mismo año, la cobertura periodística a las elecciones por la gubernatura en Jalisco. Los diarios analizados son los mismos, pero en este caso, delimita su muestra a 37 ejemplares de cada diario, seleccionados aleatoriamente del 6 de septiembre al 29 de diciembre, de los cuales 30 correspondían a la fecha previa a la jornada electoral. La muestra se conformaba con un promedio de 9 ejemplares por mes y sus unidades de análisis eran similares al estudio anterior.

Para su estudio sobre la cobertura informativa de las elecciones locales en el Estado de México, Trejo Delarbre (1991) delimita su muestra en 14 días ("entre septiembre y noviembre"), sin especificar los criterios utilizados para la selección de las fechas que integraban la muestra. Vale la pena apuntar, que para la elaboración del artículo a que se refiere este párrafo, en la que realiza un análisis comparativo de las elecciones de 1988 y 1991 , el autor utilizó los 16 días previos a la jornada electoral de la muestra utilizada para su estudio sobre las elecciones de 1988. Sin embargo, para la definición de su muestra en la investigación sobre las elecciones federales de 1991, Trejo Delarbre (2001), cuyo período abarcaba del 25 de junio al 21 de septiembre, optó por una muestra de 16 días, la cual integró mediante una especie de semana compuesta, seleccionando un día de cada semana consecutiva (lunes de la primera semana, martes de la segunda, etc), hasta completar 5 fechas, dos series de tres días seguidos (jueves, viernes y sábado) en dos semanas y finalmente otra semana compuesta de 5 
días. Para las elecciones presidenciales de 1994, cuyo período de análisis lo estableció del $\mathbf{3 0}$ de mayo al 18 de agosto, elaboró una muestra de 18 días aplicando criterios similares a la de tres años antes. Sin embargo en su estudio sobre la cobertura informativa en las elecciones para Jefe de Gobierno en el Distrito Federal en 1997, Trejo ensayó una nueva forma de integración de la muestra -una semana compuesta, una consecutiva y dos días más, para un total de 16enmarcada en el período del 28 de abril al 1 de julio.

Por parte de quien esto escribe, para el estudio sobre las elecciones presidenciales de 1994 (Aceves,2001), cuyo período de análisis abarcó del 13 de enero al 21 de agosto, se definió una muestra de 32 días para los medios escritos (3) y de 38 para noticieros televisivos (2). Para su integración se incluyeron las fechas en las que alguno de los candidatos de los tres principales partidos, realizaron actividades de campaña en la ciudad de Guadalajara, completando la muestra con fechas seleccionadas en forma aleatoria.

Daniel Hallin (1995) en su estudio sobre el comportamiento del telenoticiario 24 Horas en las elecciones presidenciales de $1994 \mathrm{el}$ estudio esta basado en un muestra de dos días de la semana seleccionados aleatoriamente de cada semana, del enero al 26 de agosto, última semana siguiente a la elección. Asimismo incluyó el día 3 de diciembre de 1993. Las dos primeras semanas de enero fueron sobremuestreadas con el objeto de permitir al análisis de la cobertura del levantamiento en Chiapas. En total se analizaron 61 días.

Una serie de trabajos que merecen mención especial, fueron los cuatro monitoreos realizados por la Academia Mexicana de Derechos Humanos en coordinación con Alianza Cívica, sobre la cobertura informativa de las elecciones presidenciales de 1994 por los noticiarios televisivos de difusión nacional: 24 Horas de Televisa y Hechos de Televisión Azteca. A los que se adicionó Enlace del Canal 11 en el último estudio. Si bien, desde 1991 diversos organismos civiles orientados hacia la observación electoral, otorgaban un sitio destacado al monitoreo de medios de comunicación, la importancia del estudio de 1994 radica, para los propósitos el presente artículo, en el tamaño de la muestra analizada, pero principalmente, en la propuesta de una "Guía para el análisis de contenido electoral en México" que con base en sus experiencias en el campo del monitoreo de medios, elaboran sus autores.

Los cuatro estudios, abarcaron en su conjunto el período comprendido del 3 de enero al 15 de julio de 1994. A excepción del 
ultimo estudio ( 1 al 15 de julio) en cuya vitrina metodológica se indica la integración de la muestra comprende a la "totalidad de las emisiones diarias", en los tres restantes se especifica que para la conformación de la misma "se grabaron casi en su totalidad las emisiones diarias de los noticiarios" (cursivas mías). La categoría de análisis fueron las noticias "que abordaron el tema de las elecciones federales, así como aquellas que tuvieron alguna relación con las mismas". Sin embargo, a pesar del carácter exhaustivo de las muestras utilizadas en los diversos reportes, en su propuesta de "Guía" la definición del tamaño de la muestra es abordado de manera harto superficial. En efecto, luego de recordar que para la determinación del período que se pretende analizar se puede optar por el censo y la delimitación de una muestra establecida mediante procedimientos estadísticos, los autores exponen que su "experiencia indica que la selección y análisis de tres períodos a lo largo de una contienda electoral (al principio, a la mitad y al final) permite obtener resultados confiables. Inclusive, si no se dispone de recursos abundantes, diez días pueden ser suficientes para establecer las inferencias sobre el papel de los medios". Así, sin mayor explicación que la de la propia "experiencia" y sin otro argumento que el referido a "que las líneas editoriales de los medios son invariables y tienden a otorgar el mismo tratamiento y espacio a los distintos actores" -de suyo cuestionablelos resultados obtenidos de tales muestreos, enfrentarían problemas respecto a su validación.

En su estudio sobre la cobertura informativa de los telenoticiarios 24 Horas y Hechos de las elecciones de 1997 para Jefe de Gobierno en el Distrito Federal, Aimée Vega (1999), señala que el período de análisis abarcó del 17 de marzo al 2 de julio, pero no especifica el tamaño de su muestra.

Finalmente, en su investigación sobre las elecciones presidenciales de 2000, José Carlos Lozano (2001) aunque enfoca la "espectacularización" del contenido informativo sobre las campañas políticas, aborda también lo relativo a la equidad en el acceso y tratamiento de los medios a los diversos candidatos. En su análisis que incluye a tres diarios nacionales y tres telenoticiarios, delimita un período comprendido del 1 de marzo al 23 de junio. Para la conformación de la muestra seleccionó "un día sí y otro no de las ediciones de los seis medios informativos", es decir un total de 57 días. Su categoría de análisis fue la nota informativa (excluyendo editoriales, cartones, buzón y columnas) aunque la conformación de 
las muestras observó características distintas para los diarios y los telenoticiarios. Mientras que en los primeros la muestra se integró con el total de las unidades de análisis, en el caso de los diarios la muestra se determino seleccionando mediante procedimientos aleatorios (de izquierda a derecha y de superior a inferior) cada tercer nota referida a la contienda electoral.

Esta revisión panorámica de los estudios sobre la cobertura informativa realizados mediante el uso del análisis de contenido y cuyas principales características se condensan en el Cuadro 1, permiten adelantar algunas conclusiones sobre los procedimientos metodológicos que los autores utilizaron en el desarrollo de sus investigaciones, específicamente en cuestiones relacionadas con la selección del objeto de estudio, la determinación del universo, la definición del tamaño de la muestra, la definición de las unidades de análisis, y la definición de la unidad de medida.

Lo que se aprecia de entrada es que, exceptuando la selección del objeto de estudio y la unidad de medida, la única característica que comparten los diferentes estudios, es su vasta diversidad. En efecto, en relación con el objeto de estudio, se aprecia una cierta consistencia en la selección de los diarios que se analizan en los estudios enfocados hacia la prensa escrita, así como en los telenoticiarios elegidos, en el caso de los medios electrónicos. Respecto a la unidad de medida, casi la totalidad de los estudios analizados se inclinan hacia la realización del análisis cuantitativo, por la adopción de una medida de tipo espacial tanto para la prensa escrita ( $\mathrm{Cms}^{2}, \mathrm{Cms} /$ columna), como para los telenoticiarios (segundos/minutos/horas). Solamente 2 autores (Aceves y Lozano) consideran además de estas unidades de tipo espacial, el número de notas como medida de cuantificación.

Las variaciones de procedimiento metodológico entre los diversos estudios, se hacen evidentes, particularmente en lo relativo a la determinación del universo y la definición del tamaño de la muestra. En el primer caso las diferencias son axiomáticas, pasan de los 348 días en los estudios de Fregoso y Arredondo a los 65 días en el estudio que Raúl Trejo realiza sobre las elecciones de 1997. A excepción de los trabajos de Trejo Delarbre (1991, 1994 y 1997) en donde sin definirlo explícitamente, determina el universo con base en los períodos establecidos por la legislación electoral para el desarrollo de las campañas políticas, no existe en los demás trabajos, una argumentación explícita que justifique el universo de análisis. Pero también son notorias las diferencias que se advierten en lo relativo a 
la definición del tamaño de la muestra. Aquí va de los 10 días sobre los que Secanella realiza su análisis, hasta los casi censos realizados por De Garay y Acosta Valverde. De nuevo, quien presenta una mayor consistencia es Raúl Trejo, que define muestras que van de los 14 a los 18 días. Empero, en el grueso de los trabajos , la relación entre la delimitación del universo y la definición del tamaño de la muestra, no establecen un procedimiento metodológico lo suficientemente riguroso que permita garantizar que los resultados obtenidos sean estadísticamente significativos.

Finalmente, en lo relativo a la definición de las unidades de análisis, aunque todos los estudios coinciden en definir a la noticia como unidad de análisis, varios autores, adicionan a ésta, otras unidades de análisis tales como los géneros de opinión, géneros interpretativos, fotografías y gacetillas, así como la ubicación de la nota.

Cuadro 1. Estudios de análisis de contenido sobre la cobertura informativa de los medios en los procesos electorales

\begin{tabular}{|c|c|c|c|c|c|c|}
\hline AUTOR & FECHA & $\begin{array}{l}\text { No. de } \\
\text { MEDIOS }\end{array}$ & UNIVERSO & $\begin{array}{l}\text { TAMAÑO DE } \\
\text { LA MUESTRA }\end{array}$ & $\begin{array}{l}\text { UNIDADESDE } \\
\text { ANALISIS }\end{array}$ & $\begin{array}{l}\text { UNIDAD DE } \\
\text { MEDIDA }\end{array}$ \\
\hline $\begin{array}{l}\text { Petra María } \\
\text { Secanella }\end{array}$ & $\begin{array}{l}1970 \\
1976 \\
1982\end{array}$ & $\begin{array}{c}3 \\
\text { Prensa }\end{array}$ & 240 dfas & $\begin{array}{c}10 \text { dras } \\
\text { Últimos dfas } \\
\text { del mes de mayo }\end{array}$ & $\begin{array}{l}\text { Primera plana } \\
\text { Columnas de } \\
\text { noticias } \\
\text { Ilustraciones }\end{array}$ & Menciones \\
\hline $\begin{array}{l}\text { Adrian de } \\
\text { Garay }\end{array}$ & 1988 & $\begin{array}{c}6 \\
\text { Prensa }\end{array}$ & 180 días & $\begin{array}{l}\text { "seguimiento" } \\
\text { de enero a junio } \\
\text { Censo } \\
\text { No especifica } \\
\text { número de dfas }\end{array}$ & $\begin{array}{l}\text { Columnas } \\
\text { de noticias }\end{array}$ & $\begin{array}{l}\text { Columnas } \\
\text { (indepen- } \\
\text { dientemente } \\
\text { de cada } \\
\text { diario) }\end{array}$ \\
\hline $\begin{array}{l}\text { Raúl Trejo } \\
\text { Delarbre }\end{array}$ & 1988 & $\begin{array}{c}6 \\
\text { Prensa }\end{array}$ & 145 dias & $26 \mathrm{dfas}(16)^{*}$ & $\begin{array}{c}\text { Notas } \\
\text { informativas, } \\
\text { Fotograflas, } \\
\text { Gacetillas }\end{array}$ & $\mathrm{cms} /$ columnas \\
\hline $\begin{array}{l}\text { Gilberto } \\
\text { Fregoso }\end{array}$ & 1988 & $\begin{array}{c}3 \\
\text { Prensa } \\
\text { (Jal) }\end{array}$ & 348 & $\begin{array}{c}66 \text { dias (56)* } \\
\text { Al azar } \\
\text { (entre } 4 \text { y } 6 \\
\text { números por mes) }\end{array}$ & $\begin{array}{c}\text { Géneros } \\
\text { informativos, } \\
\text { Géneros de } \\
\text { opinión, } \\
\text { Inserciones } \\
\text { pagadas }\end{array}$ & $\begin{array}{l}\text { Número de } \\
\text { notas } \mathrm{Cms}^{2}\end{array}$ \\
\hline $\begin{array}{l}\text { Gilberto } \\
\text { Fregoso }\end{array}$ & 1991 & $\begin{array}{c}3 \\
\text { Prensa } \\
\text { (Jal) }\end{array}$ & 114 & $\begin{array}{c}37 \text { días } \\
\text { Al azar } \\
\text { (9 ejemplares } \\
\text { por mes) }\end{array}$ & $\begin{array}{c}\text { Géneros } \\
\text { informativos, } \\
\text { Géneros de } \\
\text { opinión, } \\
\text { Inserciones } \\
\text { pagadas }\end{array}$ & $\begin{array}{l}\text { Número de } \\
\text { notas Cms }\end{array}$ \\
\hline $\begin{array}{l}\text { Pablo } \\
\text { Arredondo }\end{array}$ & 1988 & $\stackrel{2}{\text { TV }}$ & 348 & $\begin{array}{c}55(45)^{*} \\
\mathrm{Al} \mathrm{azar}\end{array}$ & Noticias & Segundos \\
\hline
\end{tabular}

' La excepción la constituyen los trabajos de Trejo Delarbre, De Garay, Acosta Valverde y Lozano. 


\begin{tabular}{|c|c|c|c|c|c|c|}
\hline Raúl Trejo & $\begin{array}{l}1991 \\
\text { D.F. }\end{array}$ & $\begin{array}{c}6 \\
\text { Prensa }\end{array}$ & & $\begin{array}{c}14(1991) \\
16 \text { (1988) } \\
\text { Al azar }\end{array}$ & $\begin{array}{l}\text { Notas } \\
\text { informativas, } \\
\text { Fotografías. } \\
\text { Gacetillas }\end{array}$ & Cms/columna \\
\hline Raúl Trejo & $\begin{array}{c}1991 \\
\text { federal }\end{array}$ & $\begin{array}{c}6 \\
\text { Prensa }\end{array}$ & 88 & $\begin{array}{c}16 \\
5 \text { dias compuesta + } \\
3 \text { dfas consecutivost } \\
3 \text { dfas consecutivost } \\
5 \text { dfas compuesta }\end{array}$ & Noticias & Cms/columna \\
\hline Raul Trejo & 1994 & $\begin{array}{c}5 \\
\text { Prensa }\end{array}$ & 81 & $\begin{array}{c}\text { 18días } \\
\text { idem } 1991\end{array}$ & Noticias & Cms/columna \\
\hline Raúl Trejo & 1997 & $\begin{array}{c}66 \\
\text { Prensa }\end{array}$ & 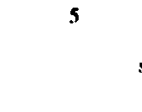 & $\begin{array}{c}16 \text { dlas } \\
\text { semana compuesta }+ \\
\text { semana consecutiva }+ \\
2 \text { dias }\end{array}$ & Noticias & Cms/columna \\
\hline $\begin{array}{l}\text { Francisco } \\
\text { Aceves }\end{array}$ & 1994 & $\begin{array}{c}3 \\
\text { Prensa } \\
2 \\
\text { TV }\end{array}$ & 220 & $\begin{array}{c}32 \text { prensa } \\
38 \mathrm{TV} \\
\text { dias seleccionados } \\
\text { actos candidato } \\
+ \text { aleatorios }\end{array}$ & $\begin{array}{c}\text { Géneros } \\
\text { informativos, } \\
\text { Interpretativos, } \\
\text { De opinion, } \\
\text { Fotograffa } \\
\text { Ubicación }\end{array}$ & $\begin{array}{c}\text { Número } \\
\text { de notas } \\
\text { Cms } \\
\text { prensa, } \\
\text { Nómero } \\
\text { de notas } \\
\text { Segundos } \\
\text { TV }\end{array}$ \\
\hline Danniel Hallin & 1994 & $\begin{array}{c}1 \mathrm{TV} \\
\text { (24 Horas) }\end{array}$ & $\begin{array}{c}258 \\
2 \text { dias semana } \\
\text { al azar }\end{array}$ & 61 & Noticias & $\begin{array}{l}\text { Segundos/ } \\
\text { minutos }\end{array}$ \\
\hline $\begin{array}{l}\text { Miguel } \\
\text { Acosta y } \\
\text { Luz Paula } \\
\text { Parra }\end{array}$ & 1994 & $\begin{array}{c}3 \\
\text { TV }\end{array}$ & 193 & Casi la totalidad & $\begin{array}{c}\text { Noticias } \\
\text { ubicación }\end{array}$ & Seg/min/horas \\
\hline Aimée Vega & 1997 & $\stackrel{2}{\text { TV }}$ & 107 & N/E & N/E & Seg/min/horas \\
\hline $\begin{array}{l}\text { Jose Carlos } \\
\text { Lozano }\end{array}$ & 2001 & $\begin{array}{l}3 \text { Prensa } \\
2 \mathrm{TV}\end{array}$ & 115 & $\begin{array}{l}\text { Dias alternos } \\
\qquad n=57\end{array}$ & $\begin{array}{l}\text { c/3er noticia } \\
\text { prensa, } \\
\text { Noticias TV }\end{array}$ & $\begin{array}{c}\text { Número } \\
\text { de notas } \\
\text { Cms }^{2} \text { prensa } \\
\text { Segundos TV }\end{array}$ \\
\hline
\end{tabular}

- Los dás al interior del paréntesis corresponden al perrodo de las campañas politicas.

\section{La delimitación del universo del objeto de estudio}

En el caso de los procesos electorales, como se puede apreciar en la revisión de los estudios, hay una cuestión que antecede al problema de la determinación del tamaño de la muestra, y éste es el de la delimitación de lo que se considera como el "universo" del objeto de estudio: más precisamente establecer una distinción entre dos conceptos frecuentemente confundidos: proceso electoral y campañas electorales.

Esta distinción que parece menor, resulta fundamental en la delimitación del universo de estudio y consecuentemente en la determinación del tamaño de la muestra. Dado a que ambos conceptos no definen de suyo los límites que establecen su temporalidad, ya que se puede argumentar, que algunos aspectos del proceso electoral aparecen como tema de opinión, en la cobertura informativa de los 
medios, con mucha antelación-Fox anuncia su búsqueda por la candidatura a la presidencia tres años antes- a las fechas establecidas para la realización de la contienda electoral. En este sentido, para el propósito del presente artículo, se optó por asumir como delimitación temporal de ambos conceptos, la establecida en la legislación electoral vigente. Así, en el caso del "proceso electoral" el período incluye desde la convocatoria que las autoridades electorales emiten para la presentación de candidaturas a determinado cargo de elección popular, las precampañas $u$ otro tipo de procedimientos partidarios mediante los cuales se eligen a los candidatos, las campañas electorales, la jornada electoral, las impugnaciones y movilizaciones que pudiesen derivarse de la jornada, hasta la validación de los resultados de los comicios por la autoridad electoral. En el caso de Jalisco la legislación establece que el proceso electoral comienza con la publicación de la convocatoria -cuya fecha límite es el 30 de enero-para las elecciones correspondientes y concluye con el dictamen y declaración de validez de la elección respectiva (Art. 227). Es decir, un período de alrededor de seis meses.

Por su parte, las "campañas electorales" de acuerdo a la legislación electoral, en el caso de Jalisco se inician "al día siguiente en que se haya declarado válido el registro para la elección respectiva" y concluyen "tres días antes de la eleccion" (Art. 65-VI). Es decir, que en Jalisco las campañas electorales presentan una duración diferente, de acuerdo al tipo de elección que se realizará. En el caso específico de la elección a la gubernatura del 2000 , sobre cuyo análisis se sustenta el presente escrito, el período establecido para las campañas electorales abarcó un lapso de 79 días comprendidos del 23 de agosto al 9 de noviembre.

\section{El tamaño de la muestra y su validación}

Para el ejercicio de validación del tamaño de la muestra se utilizaron como base los resultados del censo correspondiente a la cobertura informativa de las campañas políticas en las elecciones de 2000 para la gubernatura en Jalisco, publicada en las ediciones diarias del periódico MURAL, editado por el Grupo Reforma en la ciudad de Guadalajara, en el período que la legislación electoral establece para el desarrollo de las campañas políticas. El objetivo del censo consistió en revelar la distribución de la cobertura informativa realizada por el diario sobre las campañas electorales de los diversos candidatos. 
Se definió como nota electoral, exclusivamente a la que se relacionaba directamente con los candidatos. Se determinó incluir como unidad de análisis, el total de notas de los diversos géneros periodísticos (informativo, interpretativo y de opinión) así como las fotografías que se consideraron como un género independiente. Como unidades de medida se definieron el número de notas y los $\mathrm{Cms}^{2}$ para la cuantificación del espacio.

Se realizaron un total de 23 muestras cuya integración corresponde a diversos tipos de muestreo. Así, se hicieron 11 muestras de "semanas cronológicas" que comprendieron 77 de las 79 ediciones analizadas. Se realizaron 4 "muestras cronológicas" que se integraron mediante ediciones seleccionadas con base en intervalos de tiempo definidos cronológicamente ${ }^{3}$. Dos muestras correspondieron a un período de cada 5 días, una de cada 6 días y una de cada 3 días. También se hicieron dos muestras de "semana compuesta no probabilística" ; una integrada por 7 días y otra por 12. Así mismo se realizaron tres muestras conformadas por 14 ediciones seleccionadas mediante un procedimiento "probabilístico aleatorio simple" Finalmente, se realizaron tres muestras de "semana compuesta probabilística", una de ellas integrada por 12 días y las otras por 11 .

En todos los casos, los resultados obtenidos por las diversas muestras, se contrastaron con los resultados del censo tanto en la categoría de "número de notas" como de la categoría "espacio". Para efectos de la validación de la muestra, se consideró un resultado

2 La "semana cronológica" como su nombre lo dice se integra con las ediciones correspondientes a la secuencia cronológica de una semana, independientemente del día en que se inicie.

La "muestra cronológica" se integra con las fechas seleccionadas de acuerdo a determinados intervalos de tiempo. Así, por ejemplo, se selecciona el primer día, se establece un intervalo de días $(2,3,4)$ que se excluyen, incorporando a la muestra el siguiente día y así sucesivamente.

"Para la integración de una "semana compuesta no probabilística" se selecciona un determinado día de la primera semana (por ejemplo el lunes), entonces de la segunda semana se selecciona el día subsecuente (el martes), de la tercera semana el miércoles y así sucesivamente.

El muestreo "probabilístico aleatorio simple" se realizó mediante el procedimiento de la urna. En un recipiente se depositan el total de fechas, entonces se extraen, en forma aleatoria, el número de las mismas que se haya determinado.

'A diferencia de la no probabilística que se conforma con base en la secuencia cronológica, la "semana compuesta probabilística" se integra seleccionando mediante procedimientos probabilisticos, los días correspondientes a cada una de las semanas. 
estadísticamente significativo, aquél cuya diferencia entre los resultados de la muestra con los resultados del censo, se ajustara a los rangos de \pm 5 en todas y cada una de las variables analizadas.

Con respecto a la categoría "número de notas" se aprecia que solamente 3 de las 11 muestras de "semana cronológica" justifican su validación (Cuadro 2). En contraste todas las muestras integradas mediante una selección de fechas por lapsos de tiempo cronológicos arrojaron resultados confiables. En el caso de las "semanas compuestas no probabilísticas", ninguna de las muestras cubrió el requisito de confiabilidad. Por otra parte, solamente una de los tres muestreos realizados bajo el procedimiento "probabilístico aleatorio simple" obtuvo resultados estadísticamente significativos, mientras que dos de las tres muestras de "semana compuesta probabilística" lo hicieron(Cuadro 3).

Cuadro 2. Comparativo resultados censales y muestra semana cronológica (SC).

Número de notas

$\begin{array}{lcccccccccccc} & \text { Censo } & \text { SC1 } & \text { SC 2 } & \text { SC 3 } & \text { SC 4 } & \text { SC 5 } & \text { SC 6 } & \text { SC 7 } & \text { SC 8 } & \text { SC 9 } & \text { SC 10 SC 11 } \\ \text { PAN } & 32.2 & -5.6 & 11.4 & 2.9 & 0.5 & -3.9 & -3.1 & -1.6 & -3.3 & 2.4 & -1.1 & -3.5 \\ \text { PRI } & 38.3 & 0.5 & -10.2 & -0.8 & -5.6 & 3.4 & 0.1 & 7.0 & 8.0 & 0.9 & -7.5 & 5.7 \\ \text { PRD } & 17.2 & -1.7 & 1.4 & -1.3 & -4.8 & -4.5 & 2.5 & -7.8 & -1.2 & 7.3 & 4.7 & -1.4 \\ \text { PAN/PRI } & 2.3 & 2.3 & 0.3 & 0.1 & 2.3 & 1.1 & -10.6 & -0.2 & -1.6 & -3.0 & 0.2 & 1.5 \\ \text { RESTO } & 4.1 & 1.4 & -1.9 & -2.4 & 4.1 & 4.1 & -1.9 & 4.1 & 4.1 & -6.6 & 3.1 & -1.3 \\ \text { VARIOS } & 3.4 & 0.7 & 1.4 & 2.3 & 2.2 & 1.0 & 0.5 & -2.9 & -1.9 & -0.4 & 1.3 & -2.8 \\ \text { TODOS } & 2.6 & 2.6 & -2.4 & -0.7 & 1.4 & -1.0 & 2.6 & 1.3 & -4.0 & -0.5 & -0.5 & 1.8\end{array}$

Cuadro 3. Comparativo resultados censales y muestreos diversos.

Número de notas

\begin{tabular}{|c|c|c|c|c|c|c|c|c|c|c|c|c|c|}
\hline & Censo & $\begin{array}{c}\text { MC } \\
(5)\end{array}$ & $\begin{array}{l}\text { MC } \\
(5)\end{array}$ & $\begin{array}{r}\text { MC } \\
(6)\end{array}$ & $\begin{array}{r}\text { MC } \\
(3)\end{array}$ & $\begin{array}{c}\text { SCNP } \\
\text { (7) }\end{array}$ & $\begin{array}{c}\text { SCNP } \\
(12)\end{array}$ & MPAS & MPAS & MPS & $\begin{array}{l}\text { SCP } \\
(12)\end{array}$ & $\begin{array}{l}\text { SCP } \\
\text { (11) }\end{array}$ & $\begin{array}{l}\text { SCP } \\
\text { (11) }\end{array}$ \\
\hline PAN & 32.2 & 0.1 & 0.7 & 3.3 & 1.0 & 0.9 & -6.0 & -1.4 & -10.5 & -2.9 & -0.9 & 4.1 & 3.3 \\
\hline PRI & 38.3 & 3.9 & 0.5 & -4.0 & -0.8 & 2.4 & 8.0 & 1.4 & 6.0 & 5.6 & -1.8 & -0.5 & 1.3 \\
\hline PRD & 17.2 & 3.0 & -3.9 & -1.6 & 1.9 & -6.2 & -1.0 & 2.2 & -1.7 & 1.4 & 3.8 & -6.8 & -2.1 \\
\hline PAN/PRI & 2.3 & -2.4 & 0.9 & 1.0 & -1.2 & -0.8 & -0.1 & -1.0 & 1.1 & -3.0 & 1.7 & -0.2 & 0.1 \\
\hline RESTO & 4.1 & -2.1 & 1.2 & 2.1 & -0.6 & 4.1 & 0.5 & -1.0 & 1.1 & -0.7 & -4.2 & 4.1 & 1.9 \\
\hline VARIOS & 3.4 & -3.7 & 0.1 & 0.7 & 0.2 & -1.3 & -1.4 & -1.3 & 2.8 & 0.5 & -0.4 & 0.1 & -2.5 \\
\hline TODOS & 2.6 & 1.2 & 0.7 & -1.4 & -0.3 & 1.0 & 0.2 & 1.2 & 1.4 & -0.9 & 2.0 & -0.7 & -1.8 \\
\hline
\end{tabular}


Por su parte, la contrastación entre los resultados del censo y los diversos muestreos con base en la categoría espacio, reiteran la escasa confiabilidad de las muestras conformadas por la "semana cronológica" (solamente una de las once cubren el criterio de validación), así como la "semana compuesta no probabilística". En el caso de la "muestra cronológica", a diferencia de lo obtenido en la categoría "número de notas", cuando las 4 muestras manifestaron resultados confiables, ahora solamente la que se integró mediante el procedimiento de cada tercer día (sumando un total de 26) fue la que cubrió el requisito para su validación. Así mismo, dos de los tres muestreos realizados con el procedimiento "probabilística aleatorio simple" presentaron resultados confiables. Finalmente, dos de los tres muestreos de "semana compuesta probabilística" también presentan resultados que cumplen con el criterio establecido para reconocerles validez estadística.

Cuadro 4. Comparativo resultados censales y muestra semana cronológica (SC).

\section{Espacio}

$\begin{array}{lcccccccccccc} & \text { Censo } & \text { SC1 } & \text { SC 2 } & \text { SC 3 } & \text { SC 4 } & \text { SC5 } & \text { SC6 } & \text { SC7 } & \text { SC 8 } & \text { SC 9 } & \text { SC 10 } & \text { SC 11 } \\ \text { PAN } & 30.4 & -11.4 & 0.3 & 3.9 & -0.1 & -3.3 & -3.4 & -0.4 & 2.7 & 7.6 & -1.3 & -3.7 \\ \text { PRI } & 34.1 & 0.4 & -8.4 & -4.1 & -5.3 & 8.3 & -0.8 & 6.1 & 6.2 & 1.1 & -10.2 & 4.3 \\ \text { PRD } & 15.5 & -4.4 & 3.0 & -0.3 & -4.2 & -8.8 & 1.9 & -8.6 & 2.6 & 5.8 & 3.6 & -1.5 \\ \text { PAN/PRI } & 2.3 & 2.3 & 1.1 & -1.7 & 2.3 & 0.2 & 0.9 & -2.5 & -2.1 & -1.4 & -0.4 & 2.0 \\ \text { RESTO } & 5.3 & 4.9 & -0.4 & \mathbf{0 . 6} & 5.3 & 5.3 & -8.4 & 5.3 & 5.3 & -9.1 & 5.0 & -0.6 \\ \text { VARIOS } & \mathbf{7 . 1} & 2.9 & 2.9 & 3.9 & 3.8 & 3.3 & 4.5 & -2.0 & -7.1 & -4.4 & 6.2 & -5.5 \\ \text { TODOS } & 5.2 & 5.2 & 1.4 & -2.3 & -1.9 & -5.1 & 5.2 & 2.0 & -7.7 & 0.3 & -2.9 & 4.9\end{array}$

Cuadro 5. Comparativo resultados censales y muestreos diversos. Espacio

Censo MC MC MC MC SCNP SCNP MPAS MPAS MPS SCP SCP SCP
(5)
(5) (6)
(3)
(7) (12)
(12) (11) (11)

Censo MC (5) MC (5)MC (6) MC (3)SCNP (7)SCNP (12) MPAS MPASMPASSCP (12)SCP

\begin{tabular}{llllllllllllll} 
(11) & \multicolumn{2}{c}{$\mathrm{SCP}(11)$} & & & & & & & & & & \\
PAN & 30.4 & 0.3 & 3.3 & 1.6 & 2.5 & 1.8 & -3.2 & 0.2 & -8.9 & 0.2 & 0.3 & 2.1 & -3.0 \\
PRI & 34.1 & 5.7 & -3.4 & -5.5 & -2.4 & -0.8 & 8.3 & 2.5 & 4.1 & 2.8 & 0.1 & -1.4 & 3.7 \\
PRD & 15.5 & 0.9 & 0.0 & -0.2 & 1.4 & -9.2 & -0.9 & 1.6 & -4.8 & 1.9 & -0.3 & -5.4 & -2.2 \\
PAN/PRI & 2.3 & -2.3 & 1.0 & 1.2 & -1.7 & -3.2 & -0.1 & 0.0 & 0.5 & -3.9 & 1.3 & -1.0 & 1.1 \\
RESTO & 5.3 & -3.0 & 2.0 & 3.8 & -1.3 & 5.3 & 0.5 & -2.2 & 0.8 & -0.6 & -3.0 & 5.3 & 3.4 \\
VARIOS & 7.1 & -4.3 & -6.6 & 3.4 & 1.3 & 1.1 & -4.6 & -4.8 & 5.4 & -1.8 & -2.5 & 3.8 & -3.5 \\
TODOS & 5.2 & 2.6 & 3.5 & -4.4 & 0.2 & 4.9 & 0.0 & 2.7 & 2.7 & 1.3 & 4.1 & -3.4 & 0.4
\end{tabular}


$\mathrm{MC}=$ Muestra cronologica (cada $\mathrm{x}$ días)

SCNP= Semana compuesta no probabilistica (dfas)

MPAS = Muestra probabilistica aleatoria simple (14 dfas)

$\mathrm{SCP}=$ Semana compuesta probabilística (dfas)

\section{Conclusiones}

De lo antes expuesto, se pueden derivar algunas conclusiones respecto al problema metodológico relacionado con la integración de una muestra confiable, en la investigación de la cobertura informativa de los procesos electorales mediante el análisis de contenido.

La primera es la necesidad de precisar en forma estricta la delimitación del universo de estudio. La sugerencia es la distinción entre los conceptos de "proceso electoral" y de "campaña política", así como adoptar para su estudio los períodos cronológicos establecidos en la legislación vigente. Esto permitirá contar en el futuro con trabajos susceptibles de ser comparados entre sí, en la medida observan cierta consistencia en la delimitación del universo analizado.

La segunda es que de acuerdo a lo analizado, los tipos de muestreo que mayormente se ajustaron al criterio de validación, fueron la "muestra cronológica", específicamente la conformada por el mayor número de días $\mathrm{MC}(3)$, y la "semana compuesta probabilística". En el primer caso, merece destacarse la consistencia en resultados confiables que se observa en las "muestras cronológicas" relacionadas con la categoría de "número de notas", aunque presenta algunas incorrecciones -ciertamente menores- en lo relativo a la categoría "espacio". Lo que viene a corroborar la recomendación de José Carlos en el sentido de incluir en la muestra "el número máximo de fechas que podamos abarcar con nuestros recursos humanos, económicos y temporales". Por su parte, la "semana compuesta probabilística" se presenta como una muestra harto confiable, dos de las tres realizadas cumplen estrictamente el criterio de validación y la restante presenta distorsiones mínimas, lo que la convierte en un instrumento susceptible de ser utilizado por quien dispone de recursos limitados. Aunque vale la pena señalar, que dada la delimitación del universo de este estudio (11 semanas) el número de semanas compuestas que se pudieron conformar fue de 2 .

Es factible, entonces, obtener resultados confiables mediante el uso de un muestreo en la investigación de la cobertura informativa de los medios en los procesos electorales, a condición de realizar una delimitación estricta del universo de estudio y la definición de la 
muestra mediante los procedimientos de "muestreo cronológico" y de "semana compuesta probabilística".

\section{Bibliografía}

Aceves González, Francisco de Jesús. 2000. "La investigación académica sobre el papel de los medios de comunicación en los procesos electorales en México" en COMUNICACIÓNY SOCIEDAD Num. 37, DECS/Universidad de Guadalajara, enero-junio 2000.

Aceves González, Francisco de Jesús. 2001. LOS MEDIOS DE COMUNICACIÓN Y EL ELECTORADO TAPATÍO EN LAS ELECCIONES PRESIDENCIALES DE 1994. La construcción de la imagen pública de los candidatos presidenciales y su impacto en la percepción de los usuarios. Tesis de Doctorado. Universidad de Guadalajara

Acosta Valverde, Miguel y Luz Paula Parra Rosales. 1995. LOS PROCESOS ELECTORALES EN LOS MEDIOS DE COMUNICACIÓN. Guía para el análisis de contenido electoral en México. AMDH/ UIA, México.

Arredondo Ramírez, Pablo. 1990. «Medios de comunicación y procesos electorales. (El caso de los noticieros de televisión)» en COMUNICACIÓN Y SOCIEDAD, No. 8, enero-abril, CEICUniversidad de Guadalajara, México

De Garay y Laura Moya, Adrían. 1989. "Aproximaciones al estudios de la opinión pública y la prensa: las campañas electorales de 1988» en SOCIOLÓGICA, Año 4, Num. 11, septiembre-diciembre, UAM-Azcapotzalco,México

Fregoso Peralta, Gilberto. 1990. «Las elecciones federales de 1988 en la prensa de Guadalajara» en COMUNICACIÓN Y SOCIEDAD, Num. 8, enero abril, CEIC-Universidad de Guadalajara, México

Fregoso Peralta, Gilberto. 1991. «Cinco meses después...» en ASÍ SE CALLO EL SISTEMA, Universidad de Guadalajara, México

Hallin, Daniel C.. 1995. «Dos instituciones y un camino: Television and the State in the 1994 Mexican Election» ponencia presentada en el XIX Annual Congress of the Latin American Studies Association, Washinton, D.C., Septiembre 28-30 
Lozano, José Carlos. 1994. "Hacia la reconsideración del análisis de contenido en la investigación de los mensajes comunicacionales" en INVESTIGAR LA COMUNICACIÓN. Propuestas Iberoamericanas, Cervantes Barba y Sánchez Ruiz (coords), Universidad de Guadalajara, México.

Lozano, José Carlos. 2001. "Espectacularización en la cobertura informativa de las elecciones mexicanas a la Presidencia" en COMUNICACIÓN Y SOCIEDAD, Vol. XIV, Num. 1, Univeridad de Pamplona

Krippendorff, Klaus. 1990. METODOLOGÍA DE ANÁLISIS DE CONTENIDO. Paidós Comunicación, España.

Secanella, Petra María.1983. EL PERIODISMO POLÍTICO EN MEXICO. Editorial Mitre, España.

Trejo Delarbre, Raúl. 1990. «Campaña y elecciones en la prensa de la ciudad de México» en COMUNICACIÓN Y SOCIEDAD, No. 8, enero abril CEIC-Universidad de Guadalajara, México. También en ASI SE CALLO EL SISTEMA, Universidad de Guadalajara, México

Trejo Delarbre, Raúl. 1991. "La prensa y los partidos" en NEXOS Num. 164, agosto, México

Trejo Delarbre, Raúl. 2001. MEDIOCRACIA SIN MEDIACIONES. Prensa, televisión y elecciones. Cal y Arena, México.

Vega, Aimée. 1999. "El proceso electoral de 1997: los noticiarios en pantalla, la sociedad ante la pantalla" en UNIVERSIDAD DE MÉXICO Vol. LIV, Num. 582-583, julio-agosto, UNAM, México 